Revista Brasileira do Esporte Coletivo - v. 4. n. 3. 2020.

ARTIGO ORIGINAL

\title{
ANALISE DESEMPENHO ESPORTIVO NO HANDEBOL DURANTE O CICLO MENSTRUAL NAS FASES FOLICULAR E OVULATÓRIA
}

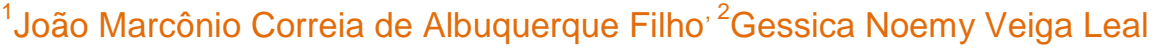

\begin{abstract}
RESUMO
Introdução: A inclusão de mulheres no esporte e oscilações hormonais decorrentes dos aspectos fisiológicos do sistema reprodutor feminino, o ciclo menstrual é citado em literaturas como fator determinante no ponto negativo e positivo em indivíduos atletas. Objetivo: analisar o desempenho esportivo em atletas de Handebol durante o ciclo menstrual, nas fases folicular e ovulatória. Método: 14 atletas de handebol entre 18 e 35 anos, foram submetidas ás avaliações com bioimpedância octopolar InBody 720, composição corporal. Força de membros superiores através da preensão manual com dinamômetro de mão mecânico. À força explosiva (potência) de membros inferiores utilizou-se Counter Movement Jump (CMJ) com auxílio dos braços. O nível de significância foi estabelecido em $\mathrm{p}<0,05$, para comparação dos dados, analisados no pacote estatístico Statistical Package for the Social Science V.21. Resultados: Para composição corporal na fase folicular e ovulatória; o índice de massa

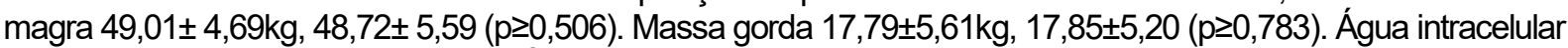
$22,43 \pm 2,17,22,90 \pm 2,73(p \geq 0,300)$. Água extracelular $13,42 \pm 1,32,15,45 \pm 7,77(p \geq 0,350)$, e Porcentagem de Gordura 26.26 $\pm 5,79,27,08 \pm 5,97$ ( $p \geq 0,188$ ). No desempenho físico podemos observar os resultados da força em potência em membros inferiores 1572,41 $\pm 94,81,1695,50 \pm 237,05$, ( $p \geq 0,066$ ). Potência w/kg 26,54 $\pm 5,32$, $25,33 \pm 1,61$, ( $p \geq 0,455)$. Tempo no ar 516,08 $\pm 28,07,526,58 \pm 33,53,(p \geq 0,136)$. Membros superiores mão direita $37,16 \pm 4,44,38,04 \pm 4,57$ ( $p \geq 0,375)$. Mão esquerda 33,37 $\pm 5,67,33,87 \pm 5,54$, ( $p \geq 0,724)$. Conclusão: Os dados constataram que não houve alternância, mantendo uma constância na composição corporal e nos níveis de forca em membros superiores, potência de membros inferiores e não havendo influência no desempenho das atletas, mantendo um nível padrão das variáveis nas duas fases do ciclo menstrual.
\end{abstract}

Palavras-chave: ciclo menstrual, fase folicular, fase anulatória, desempenho, handebol.

\section{Analysis of sports performance in handball during the menstrual cycle in the follicular and ovulatory phases}

\section{ABSTRACT}

Introduction: Due to the inclusion of women in sports and hormonal oscillations due to the physiological aspects of the female reproductive system, the menstrual cycle is cited in literature as a determinant of negative and positive point in athletes individuals. Objective: To analyze the sporting performance in Handball athletes during the menstrual cycle in the follicular and ovulatory phases. Method: 14 handballs athletes aged between 18 and 35 years underwent evaluations made with octopolar bioimpedance InBody 720 , to analyze body composition. Upper limb strength was measured by manual gripping with a mechanical hand dynamometer. The evaluation of the explosive strength (power) of lower limbs was used the technique of Counter Movement Jump (CMJ) with the aid of the arms. The level of significance was established in $p<0.05$, for comparison of the data, analyzed in the statistical package Statistical Package for Social Science (SPSS) version 21.0. Results: For body composition in the follicular and ovulatory phases; the lean mass index $49.01 \pm 4.69 \mathrm{~kg}, 48.72 \pm 5.59$ $(p \geq 0.506)$. fat mass $17.79 \pm 5.61 \mathrm{~kg}, 17.85 \pm 5.20(p \geq 0.783)$. intracellular water $22.43 \pm 2.17,22.90 \pm$ $2.73(p \geq 0,300)$. extracellular water $13.42 \pm 1.32,15.45 \pm 7.77(p \geq 0.350)$, and fat percentage $26.26 \pm$ $5.79,27.08 \pm 5.97(p \geq 0.188)$. In physical performance; we can observe the results of the power in the lower limbs 1572,41 $\pm 94,81,1695,50 \pm 237,05,(p \geq 0,066)$. Power $w / k g ~ 26.54 \pm 5.32,25.33 \pm 1.61$, $(p \geq 0.455)$. Air time 516.08 $\pm 28.07,526.58 \pm 33.53,(p \geq 0.136)$. Upper limbs right hand $37.16 \pm 4.44$, $38.04 \pm 4.57,(p \geq 0.375)$. Left hand $33.37 \pm 5.67,33.87 \pm 5.54,(p \geq 0.724)$. Conclusion: the data obtained showed that there was no alternation maintaining a constancy in the body composition and in the levels of strength in the upper limbs, power of lower limbs and there being no influence on the performance of the athletes maintaining a standard level of the variables in the two phases of the menstrual cycle.

Key words: menstrual cycle, performance, handball. 
Endereço para correspondência:

Prof. Me. João Marconio Correia de Albuquerque Filho

Rua Vereador Severino Rodrigues, 81- Bairro: Municípios

Santa Rita-PB

CEP - 58303-350

e-mail: marconiohand@hotmail.com

\section{INTRODUCÃ̃O}

De acordo com Power (2014), o envolvimento de mulheres em esportes de competição aumentou de forma significativa ao longo das últimas três décadas. Anteriormente, muitas das decisões relativas à participação de mulheres nos esportes e programas de exercício eram tomadas com base e informações fisiológicas limitadas ou inexistentes. Perante a vasta integração social introduzindo classes sociais e sexo, o índice de inclusão do sexo feminino no meio esportivo vem aumentando cada vez mais. Mulheres em idade reprodutiva apresentam alterações hormonais importantes relacionadas às fases do ciclo menstrual (CM). O conjunto de sintomas emocionais e físicos que decorrem das fases do $\mathrm{CM}$, podem afetar negativamente as atividades de mulheres, $O$ ciclo normalmente varia de 21 a 35 dias, com média de 28 dias, podendo ser dividido em três fases distintas: folicular, ovulatória e lútea.

Para Constanzo (2013), o período folicular do ciclo menstrual está presente durante $01^{\circ}$ ao $14^{\circ} \mathrm{dia}$, onde ocorre 0 desenvolvimento do folículo primordial até o estágio de folículo de De Graaf. Os receptores do hormônio luteinizante (LH) e hormônio folículo estimulante (FSH) são regulados nas células da teca e da granular, os níveis de estradiol aumentam causando proliferação do útero. Os níveis desses hormônios são suprimidos através da retroalimentação negativa do estradiol sobre a adeno-hipófise. Diminuem os níveis de progesterona. $\mathrm{Na}$ fase ovulatória acontece no $14^{\circ}$ dia antes da menstruação, em um ciclo de 28 dias. No final da fase folicular, a síntese de estradiol exerce um efeito de retroalimentação positiva sobre as secreções dos hormônios FSH e LH, na ovulação ocorre pico de LH induzido pelo estrogênio diminuindo após ovulação, aumentando na fase lútea na síntese de estrogênio e progesterona, a partir do $14^{\circ} \mathrm{a} 28^{\circ}$ dia.
Sobre os hormônios de estrogênio e progesterona: (1) baixo estrogênio e baixa progesterona estão presentes durante a fase folicular, (2) estrogênio alto e baixos níveis de progesterona estão presentes durante a fase ovulatória, e (3) estrogênio elevado e altos níveis de progesterona estão presentes durante a fase lútea (NAAMA e COL., 2005). Os hormônios gonadotróficos são estimulados e controlados através de um hormônio liberador hipotalâmico, chamado GRNH, e ambos são destinados aos órgãos alvos, que estão diretamente ligados aos níveis dos hormônios ovarianos. As oscilações hormonais, durante o ciclo menstrual, atraem especulações de estudiosos na busca de conhecimentos sobre o comportamento fisiológico das mulheres durante as fases do ciclo menstrual, em relação ao desempenho em suas práticas físicas.

Alguns estudos apontam variações nas capacidades físicas do sexo feminino devido às fases do ciclo menstrual (REDMAN, WEATHERBY, 2003; DRAKE et al.; 2004). Estes autores referenciam que tais oscilações endócrinas podem afetar o desempenho na prática de exercícios (apud DIAS,2005). A secreção de progesterona está associada à termorregulação corporal, promovendo aumento da temperatura basal do corpo e vasodilatação periférica, propiciando maior fluxo sanguíneo, induzindo à transpiração. Esse aumento da excreção de água e sódio pelos rins pode provocar aumento nas concentrações de aldosterona e consequente aumento do hormônio antidiurético (ADH), contribuindo para maior retenção de fluídos (LEBRUN; RUMBALL, 2001; apud DRUCIAK, 2015).

Os estrogênios também atuam no armazenamento de glicogênio, tanto hepático, quanto muscular e pelo aumento da síntese lipídica, orientando o metabolismo para uma maior utilização de ácidos graxos e glicerol, em detrimento dos glicídios, fazendo com que 
a mulher possua valores de lactato mais baixos em resposta ao exercício físico (ASHLEY et al., 2000; apud DRUCIAK, 2015). Soares et al., (1984); Gonçalves et al., (1991); Glaner e Pires Neto (1997); Cunha Júnior (2002), destacam a carência de pesquisas com 0 handebol no Brasil. $O$ desenvolvimento do atleta é determinado, dentre outros fatores, pelo conhecimento das características inerentes ao desporto por ele praticado, fator este que parece propiciar a aplicação adequada de estratégias que têm influência em seu melhor rendimento (MEDINA, 2000; apud JUNIOR, 2006). O handebol como modalidade esportiva coletiva, tendo os atletas, com grande parte do jogo em constante movimentação dentro de quadra no ataque ou defesa, a velocidade, a agilidade a força e a potência são qualidades físicas amplamente utilizadas durante a partida, com uma constante troca de direção, ataque, defesa, contra-ataque, arremessos, dribles e fintas.

As adaptações fisiológicas causadas por um determinado programa de treinamento

\section{MÉTODO}

O referido estudo trata-se de uma pesquisa de campo, de natureza préexperimental, quantitativa, do tipo descritiva e de caráter transversal (THOMAS, NELSON, SILVERMAN, 2012). A amostra do estudo fora constituída por 12 atletas de handebol de quadra, da equipe UNIPÊ, do gênero feminino, que participam da equipe de handebol por pelo menos 1 ano, com idade entre 18 e 35 anos, possuindo ciclo menstrual regular de 28 a 31 dias, sem lesões osteomioarticulares em membros superiores e inferiores. As atletas que se lesionaram durante a realização do estudo, que estavam com alguma doença relacionada ao sistema reprodutor feminino (síndrome ovário, poli cistos, endometriose ou mioma), foram excluídas da pesquisa.

O projeto foi submetido ao Comitê de Ética em Pesquisa do Centro Universitário de João Pessoa (UNIPÊ). Após sua aprovação pelo Comitê, com o número do CAAE: 87391018.6.0000.5176, todas as participantes leram e assinaram o termo de consentimento livre e esclarecido e a pesquisa conforme a Resolução 466/12 - Conselho Nacional de Saúde (CNS) para experimentos envolvendo humanos.

Para a análise comparativa, as atletas foram avaliadas durante as duas fases do ciclo menstrual, aproximadamente no mesmo horário. resistido são específicas a ele. Desse modo, o melhor programa de treinamento a ser aplicado para aprimorar a realização de uma tarefa específica, tal como um determinado esporte ou atividade física, é aquele que simula as características da atividade física. A periodização, além de propiciar maiores ganhos em força e potência, quando comparada a modelos não-variados, segundo a maioria dos estudos, também é responsável por um maior aumento de massa livre de gordura, hipertrofia muscular e decréscimo na porcentagem de gordura corporal (KRAMER, 2009).

O presente estudo tem como objetivo analisar o desempenho físico das atletas de handebol durante 0 ciclo menstrual nas fases folicular e ovulatória.

A primeira avaliação foi realizada entre - $1^{\circ}$ e $\circ 3^{\circ}$ dia após fluxo menstrual (fase folicular); o segundo, entre o $10^{\circ}$ e o $14^{\circ}$ dia (fase ovulatória). Foi realizado para a mensuração da composição corporal (teste de bioimpedância) e os testes de desempenho dos Membros Superiores: Preensão Manual (força) e potência de Membros Inferiores: salto vertical.

O levantamento dos dados foi realizado no laboratório de fisiologia do exercício do curso de educação física, do Centro de Ensino Universitário de João Pessoa (UNIPE), localizado na $\mathrm{Br} 230, \mathrm{~km} 22$, s/n, Água Fria - João Pessoa/PB.

\section{Instrumentos e procedimentos:}

$\mathrm{Na}$ avaliação de composição corporal para mensurar a massa corporal, índice de massa corporal, massa de músculo esquelético, massa gorda, percentual de gordura, água intracelular e água extracelular foi utilizada a bioimpedância (Octopolar InBody 720), cumprindo os procedimentos especificados na literatura. As atletas receberam instruções para realização desta avaliação, tais como: a) estar em jejum, b) não consumir bebidas alcoólicas 48 horas antes do exame, c) não realizar exercícios físicos com intensidade 12 horas antes da avaliação, d) não utilizar objetos metálicos durante o exame, 


\section{Desempenho e ciclo menstrual em atletas de handebol}

e) realizar a avaliação com trajes de banho ou roupa interior (PITANGA et al., 2012).

$O$ teste de força de membros superiores foi feito através da preensão manual de um dinamômetro de mão mecânico, marca TAKEI, modelo SMEDLEY III T-18A.com capacidade máxima de $100 \mathrm{kgf}$. As avaliadas do estudo permaneceram em posição bípede, estando com o ombro em posição neutra, cotovelo em extensão e punho em posição neutra (intermediária entre pronação e supinação). Antes de realizar a dinamometria, todas as participantes foram orientadas quanto à execução, familiarizandose com o equipamento, para evitar comprometimentos dos resultados, devido ao processo de adaptação. Foram realizadas três tentativas em cada mão, sendo considerado o maior valor de preensão manual obtido no teste, para se obter a força de preensão manual de forma alternada, iniciando com o lado direito, com duração de cinco segundos e intervalo de um minuto entre uma aferição e outra. Dado forma de incentivo verbal (vai, vai, força, força) em que o indivíduo pressiona 0 aparelho quando o mesmo imitará um sinal sonoro após a contagem regressiva de três segundos.

Para avaliação da força explosiva (potência) de membros inferiores utilizou-se a técnica de (Counter Movement Jump), salto vertical, com auxílio dos braços, conforme

\section{RESULTADOS}

Nesta pesquisa foram inscritas $(n=14)$ atletas de handebol de quadra, com faixa etária entre 18 aos 35 anos de idade, nas quais foram feitas as coletas em $(n=12)$, tendo duas exclusões do total, decorrentes de motivos pessoais. As participantes fizeram todo procedimento de acordo com a programação das datas referentes aos períodos folicular e ovulatória.

Estudos enfocaram a composição e a melhor maneira de medir seus vários componentes, metodologia que divide em dois compartimentos distintos: (1) peso (massa) corporal isenta de gordura e (2) peso massa de gordura, outros estudos subsequentes sobre composição corporal ampliam o modelo protocolo proposto por Bosco (2007), em que o atleta fica em pé com o tronco ereto e joelhos em extensão a $180^{\circ}$, realizando o salto vertical com o contra movimento. A flexão do joelho acontece aproximadamente no ângulo de $120^{\circ}$, em seguida o executante faz a extensão do joelho, procurando impulsionar o corpo para o alto e na vertical. Os joelhos devem permanecer em extensão durante a fase de voo e o intervalo entre tentativas é de dez segundos. Três tentativas foram realizadas, sendo considerada a melhor marca e, para análise dos saltos, foi utilizada plataforma de contato (CEFISE®), conectada ao sistema para medida de salto (Jump System®).

\section{Análise estatística:}

Os dados estão apresentados através de estatística descritiva. A amostra deste estudo apresenta distribuição normal pelo teste de Shapiro-Wilk, atendendo aos pressupostos de normalidade, foi utilizado o teste $\mathrm{t}$ pareado para a análise de possíveis diferenças significativas através do desempenho esportivo nas atletas durante as fases do ciclo menstrual. Foi adotado o nível de significância de $p<0,05$. Os dados foram analisados no pacote estatístico computadorizado Statistical Package for the Social Science (SPSS) versão 21.0.

de dois componentes, de forma a incluir a variabilidade biológica em três (água, proteína, gordura) ou quatro (água, proteína, mineral ósseo, gordura) (MCARDLE, 2016).

Através desse princípio, em termos anatômicos, a composição corporal pode ser mensurada trazendo resultados precisos deste, através da massa magra, referente a músculos total e gorda total ao tecido adiposo, como, além disso, ao mesmo tempo engloba os elementos biológicos relacionados a este estudo como osmolaridade celular, volume de líquido intracelular que atribui $40 \%$ do peso corporal equivalente a dois terços da (ACT), e extracelular $20 \%$, estando correlacionado a um terço da água corporal total. 
Desempenho e ciclo menstrual em atletas de handebol

Tabela 1. Caracterização das Atletas - Composição corporal no Período folicular (menstrual) e Período ovulatória.

\begin{tabular}{cccc}
\hline Composição Corporal & $\begin{array}{c}\text { Período Folicular } \\
\text { (Menstrual) }\end{array}$ & $\begin{array}{c}\text { Período } \\
\text { Ovulatório }\end{array}$ & $\mathbf{p < 0 , 0 5}$ \\
\hline Peso (kg) & $66,72 \pm 7,98$ & $66,81 \pm 8,30$ & 0,912 \\
\hline Massa Magra (kg) & $49,01 \pm 4,69$ & $48,72 \pm 5,59$ & 0,506 \\
\hline Massa Gorda (Kg) & $17,79 \pm 5,61$ & $17,85 \pm 5,20$ & 0,783 \\
\hline Percentual Gordura (\%) & $26.26 \pm 5,79$ & $27,08 \pm 5,97$ & 0,188 \\
\hline Água Intracelular (I) & $22,43 \pm 2,17$ & $22,90 \pm 2,73$ & 0,300 \\
\hline Água Extracelular (I) & $13,42 \pm 1,32$ & $15,45 \pm 7,77$ & 0,350 \\
\hline
\end{tabular}

Como demonstrado na tabela 1, à caracterização da amostra, utilizou a bioimpedância em que se obtiveram os resultados das fases foliculares, peso; $(66,72 \pm 7,98 \mathrm{~kg})$, índice de massa magra; $(49,01 \pm 4,69 \mathrm{~kg})$, massa gorda $(17,79 \pm 5,61 \mathrm{~kg})$, água intracelular $(22,43 \pm 2,17 \mathrm{~L})$, água extracelular $(13,42 \pm 1,32 \mathrm{~L})$, e porcentagem de gordura (26.26 $\pm 5,79 \%)$. Na fase ovulatória 0 peso $(66,81 \pm 8,30)$, índice de massa magra $(48,72 \pm 5,59)$, massa gorda $(17,85 \pm 5,20)$, água intracelular $(22,90 \pm 2,73 \mathrm{~L})$, água extracelular $(15,45 \pm 7,77 \mathrm{~L})$, e porcentagem de gordura $(27,08 \pm 5,97)$.

Tabela 2. Potência (w), Potência (w/kg), tempo ar, força membros superiores nos períodos folicular e ovulatório.

\begin{tabular}{cccc}
\hline Variáveis & $\begin{array}{c}\text { Período Folicular } \\
\text { (Menstrual) }\end{array}$ & Período Ovulatório & p>0,05 \\
\hline SV Potência $(\mathbf{w})$ & $1572,4 \pm 94,81$ & $1695,50 \pm 237,05$ & 0,066 \\
\hline SV Potência $(\mathbf{w} / \mathbf{k g})$ & $25,33 \pm 1,61$ & $26,54 \pm 5,32$ & 0,455 \\
\hline SV Tempo no AR $(\mathbf{m s})$ & $516,08 \pm 28,07$ & $526,58 \pm 33,53$ & 0,136 \\
\hline Força PM Dir $(\mathbf{k g})$ & $37,16 \pm 4,44$ & $38,04 \pm 4,57$ & 0,375 \\
\hline Força PM Esq. $\mathbf{( k g )}$ & $33,37 \pm 5,67$ & $33,87 \pm 5,54$ & 0,724 \\
\hline
\end{tabular}

$\mathrm{Na}$ tabela 2 podemos observar os resultados da força em potência em membros inferiores $(1572,41 \pm 94,81 \mathrm{w})$, potência $\mathrm{w} / \mathrm{kg}$ $(26,54 \pm 5,32 \mathrm{w} / \mathrm{kg})$, tempo no ar $(516,08 \pm$ 28,07ms), membros superiores mão direita $(37,16 \pm 4,44 \mathrm{~kg})$, mão esquerda $(33,37 \pm$ $5,67 \mathrm{~kg}$ ), na fase folicular e na fase ovulatória obtivemos os resultados da potência em membros inferiores (1695,50 \pm 237,05),
Para Hall (2014) potência muscular é o produto entre força muscular e velocidade de encurtamento muscular. A potência apresenta a velocidade com que o trabalho é realizado, e é importante porque a taxa de trabalho ou débito de potência identifica intensidade do exercício (POWER, 2014). A potência pode ser apresentada como a energia exercida que ao sair da inércia, capaz de produzir o movimento gerando uma força explosiva, em contrapartida envolvendo uma velocidade máxima. 
Desempenho e ciclo menstrual em atletas de handebol

\section{DISCUSSÃO}

O handebol é um desporto em que a modalidade atua com predominância 0 sistema anaeróbico e exige um bom rendimento e desempenho físico dos atletas em suas valências físicas determinantes. Jogadores desse desporto, necessariamente, utilizam as variáveis: força de explosão, potência, agilidade, velocidade. $O$ referido estudo teve como finalidade mensurar os níveis das capacidades físicas, através da força e potência, das atletas de handebol de quadra em dois momentos: fase folicular e ovulatória. Jonge (2003) e Constatini, Dubnov e Lebrun (2005) destacam alterações fisiológicas e no desempenho das capacidades físicas durante o ciclo menstrual como.

Fase Menstrual: período em que ocorre maior incidência de instabilidade emocional e irritabilidade. Nota-se que nessa fase há redução da força, velocidade e resistência; Fase Ovulatória: diminuição da capacidade de coordenação e força (DOUGLAS apud DRUCIAK, 2015).

Considerando as oscilações de níveis hormonais como principal influência na alteração composição corporal do sexo feminino durante o ciclo menstrual as fases foliculares e ovulatória, enfatizando (peso, massa magra, massa gorda, líquido intracelular, líquido extracelular e porcentagem de gordura), independente de usos de contraceptivos, em nossa análise obtivemos tais resultados como demonstrado na tabela 1. O peso com significância de $(p<0,912)$ Massa Magra $(p<0,506)$, massa gorda $(p<0,783)$, gordura \% $\quad(p<0,188)$. Líquido intracelular $(p<0,300)$, líquido extracelular $(p<350)$. Em decorrência aos resultados da nossa amostra, observou-se que não houve diferença significativa entre as fases do ciclo menstrual, constatando que não há influência do ciclo menstrual no desempenho físico. Em relação as variáveis de potência de salto com valor $(p<0,066)$ potência em $\mathrm{kg}(p<0,455)$, tempo no ar $(p<0,136)$. Força de membros superiores preensão manual mão direita $(p<0,375)$, mão esquerda $(p<0,724)$. Corroborando com outros estudos, no referido estudo não apresentou resultados estatisticamente significativos na composição corporal, como também no desempenho esportivo das atletas, durante as duas fases do ciclo menstrual.

Um estudo feito com 10 atletas de natação usuárias de contraceptivos orais das categorias Sênior e Júnior (18,7 $\pm 1,6$ anos, $64,4 \pm 5,4 \mathrm{~kg}, 165,0 \pm 0,0 \mathrm{~cm}$ ), analisou a força muscular e percepção subjetiva do esforço (PSE). As atletas realizaram, de forma aleatória, sendo uma em cada fase do CM, em três sessões experimentais: folicular ( $1^{\circ}$ ao $4^{\circ}$ dia do $\mathrm{CM}$ ), ovulatória $\left(12^{\circ}\right.$ ao $\left.15^{\circ} \mathrm{dia}\right)$ e lútea ( $21^{\circ}$ ao $27^{\circ}$ dia). A força muscular foi avaliada pelo teste de dez repetições máximas (10RM) nos exercícios leg-press $45^{\circ}$, puxada pela frente, agachamento livre e supino reto. A PSE foi verificada através da escala OMINI-RES ( 0 a 10). Os resultados demonstraram que a carga para $10 \mathrm{RM}$, na puxada pela frente, foi maior na fase ovulatória $(33,0 \pm 2,6 \mathrm{~kg}) \mathrm{em}$ comparação a fase folicular $(30,5 \pm 1,6 \mathrm{~kg})(\mathrm{p}$ $<0,05)$. No agachamento houve diferença entre as fases folicular $(70,0 \pm 20,7 \mathrm{~kg})$ e lútea $(78,3 \pm 20,5 \mathrm{~kg})(p<0,05)$. Para a PSE, houve diferença apenas no supino reto, entre as fases folicular e lútea $(7,8 \pm 1,3$ vs. $9,0 \pm 1,3$, respectivamente, $p<0,05)$. Com isso, concluiu-se que as diferentes fases do CM podem modificar a força muscular e a PSE em atletas de natação que utilizam contraceptivos orais (FORTES e COL., 2015).

Tal estudo mostrou que houve diferenças de força entre as fases folicular e ovulatória nas atletas de natação, podendo estar relacionada à dosagem de hormônios, com o uso de C. O. Já na referida investigação, não foi encontrada diferença significativa nas variáveis de forças em membros superiores entre essas duas fases, independente do uso de contraceptivos.

O estudo de caso de Morais e col. (2008) verificam a relação do treinamento de tênis de campo com o ciclo menstrual em uma atleta com 17 anos de idade. O período de treinamento foi de 16 semanas. As avaliações motoras foram realizadas em dois momentos, no período de ovulação do ciclo menstrual da atleta investigada. Foram analisadas as capacidades motoras específicas ao tênis de campo; velocidade, potência muscular de membros superiores, potência muscular de membros inferiores e agilidade. Entretanto, as outras variáveis, massa corporal, IMC e percentual de gordura tiveram diminuição, os valores, respectivamente, (6,0; 5,9 e 10,4\%). A potência muscular de membros inferiores e superiores, demonstrado nos testes de potência, alterou-se positivamente em 16,1\% para as pernas e $23,6 \%$, para os membros superiores. Os resultados encontrados demonstram que houve melhora considerável em todas as variáveis, sugerindo um planejamento de treino baseado na individualidade biológica.

Os resultados encontrados nesta pesquisa durante os períodos folicular, a partir do $1^{\circ}$ dia da menstruação ao $3^{\circ}$, e no $14^{\circ}$ dia referidamente ao período ovulatório, 
mensuração à respeito da composição corporal nas atletas, apesar desses valores serem superiores ao da pesquisa acima citada, deu-se que, o valor no índice de massa magra $(p=0,506)$, massa gorda $(p=0,783)$, porcentagem de gordura $(p=0,188)$, em evidência aos dados, estatisticamente torna-se claro que não ocorrem oscilações da composição corporal durante os dois períodos do ciclo menstrual. Como também demonstrado na tabela 2, não houve alterações nos testes de força e potência. As alterações no desempenho da atleta podem estar ligadas às respostas fisiológicas dos estímulos, em que obteve microciclos durante 16 semanas para um novo teste e relata um desconforto nos testes durante as fases menstruais, sugerindo especulações se a atleta estaria em um período competitivo entre essas fases.

No estudo da revista eletrônica Saúde e Ciência (RESC), 18 mulheres jogadoras de futebol foram avaliadas para amostra, com idade entre 23 anos e índice de massa corpórea de 18,5 e $24,9 \mathrm{~kg} / \mathrm{m}^{2}$, feitas avaliações antropométricas para aferir peso, estatura e índice de massa corpórea, realizado em duas etapas, durante o período menstrual e outra logo após quatorze dias do ciclo menstrual, análise de potência através de um teste Runing based anaerobic Sprint (RAST), 6 corridas de 35 metros e 10 segundos de intervalos. Através desse trabalho concluiu-se que não houve alterações significantes no desempenho físico das atletas profissionais de futebol, mantendo a potência ( $\mathrm{PMi}=5.04 \pm 0.4$ vs. $4,24 \pm 0.1 \mathrm{P}=0.56$, Pme $=6.37 \pm 0.1$, vs. $5.45 \pm 0.3 \mathrm{P}=0.48$, e $\mathrm{PMa}=8,04 \pm 0.1$, vs. $6.73 \pm 0.2$ unidades, $P=0.50$ ), como também as medidas antropométricas pesos $(60.8 \pm 0.3$, vs. $61.9 \pm 0.1 \mathrm{Kg}, \mathrm{P}=0.46)$ e $(\mathrm{IMC}=22.2 \pm 0.3$, vs. $21.7 \pm 0.1 \mathrm{Kg}, \quad \mathrm{P}=0.42$ ), com resultados semelhantes durante e pós ciclo menstrual (CARVALHO, 2014).

Com base no fato que a fase folicular inicia a partir do primeiro dia da descamação do endotélio (menstruação). Há uma margem de erro no $14^{\circ}$ dia da ovulação, podendo ocorrer entre $\circ 12^{\circ}$ e $16^{\circ}$ dia, tais resultados da fase folicular e ovulatória foram semelhantes aos achados desta pesquisa, assim possibilitando uma maior confiabilidade aos resultados deste estudo, incluindo índice de massa corporal e potência, a partir de nossos resultados. Em relação à potência de membros inferiores, os resultados da referida amostra foram superiores às atletas do estudo de Carvalho.

Segundo Teixeira (2018), a análise da composição corporal nos períodos do ciclo menstrual, fases folicular, ovulatória e lútea, de 44 estudantes universitárias, entre 18 e 35 anos, um grupo $(n=24)$ utilizava regularmente contraceptivos hormonais e outro grupo (n $=20$ ) não utilizava contraceptivos, cada grupo fez testes de avaliação em cada fase do ciclo menstrual; foram mensurados valores antropométricos de peso, estatura e circunferências de tórax, cintura, abdômen e coxa. Para a composição corporal, foi estimado o percentual de gordura e a massa livre de gordura (MLG). Os resultados não demonstraram diferenças significativas IMC $(\mathrm{kg} / \mathrm{m} 2) 21,1 \pm 1,621,1 \pm 1,5$ intra, e IMC $(\mathrm{kg} / \mathrm{m} 2) 22,9 \pm 3,722,8 \pm 3,8$ intergrupos $e$ para todos os valores analisados entre as diferentes fases. Em conclusão, parece que o CM não altera a composição corporal e as medidas antropométricas de universitárias, independente do uso de contraceptivos hormonais.

À conotação feita do estudo, notou-se que os resultados associados foram parecidos com os do referido estudo, no qual também não sucedeu alterações na composição corporal (percentual de gordura e massa magra). Corroborando com esta pesquisa de dados, tendo em vista que a nossa pesquisa foi feita com atletas e o estudo acima não relata se as participantes eram atletas ou praticantes de exercícios físicos.

De Acordo com Druciak (2015), estudos comparativos feitos em 10 mulheres, de vinte a trinta anos de idade, nas fases de menstruação e ovulatória, com o ciclo menstrual regular, com uso de contraceptivos, sem limitações físicas e praticantes de treinamento resistido, nas quais foram feitos

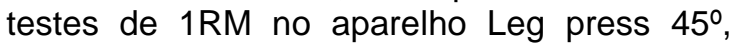
durante calendários individuais, o valor médio da força muscular do grupo avaliado no teste durante a fase menstrual foi $240 \pm 46,7 \mathrm{~kg} \mathrm{e}$ durante a fase ovulatória foi $253 \pm 56,9 \mathrm{~kg}$, mostraram resultados estatisticamente significativos entre as duas médias nos níveis de força das mulheres avaliadas.

No referido estudo, o valor médio alcançado no período folicular sobre a força em membros inferiores, em forma de potência $(w / \mathrm{kg})$, foram $26,54 \pm 5,32 \mathrm{~kg}$, e na fase ovulatória $25,33 \pm 1,61 \mathrm{~kg}$, resultando em $(p=0,455)$, não havendo valor significativo nas duas fases. Não houve semelhança nos resultados com tal estudo, em que obteve alteração de força, entre as fases avaliadas supondo-se que deveria estar associado ao tipo de estímulo já que as mulheres já eram praticantes de treinamento resistido.

Em outra investigação foram avaliadas 11 mulheres, para avaliação da força muscular 
Desempenho e ciclo menstrual em atletas de handebol

e foram aplicados o teste de 10RM nos exercícios leg press e remada sentada na polia baixa e teste de força de preensão manual. Somado a isso foram analisados os sinais e sintomas decorrentes das fases do CM e percepção subjetiva de esforço (PSE) e recuperação (PSR).

Os resultados do estudo envolvendo o ciclo menstrual e o desempenho físico demostram que maiores sintomas na fase menstrual e alteração nos níveis de força para o teste de 10RM, leg press $(59,0 \pm 13,6 \mathrm{~kg}$ vs $62,6 \pm 11,8 \mathrm{~kg}$ ) remada sentada na polia baixa $(42,6 \pm 6,4 \mathrm{~kg}$ vs $45,7 \pm 7,8 \mathrm{~kg})$, respectivamente com maiores valores na fase pós-menstrual. Para força de preensão manual e PSR não foram observadas diferenças significativas. Com isso, conclui-se que para a variável de força dinâmica o CM é capaz de alterar a força

\section{CONCLUSÃO}

A partir dos resultados deste estudo, confirma-se que não há alterações nas variáveis em que as atletas de handebol de quadra foram avaliadas, sem diferença significativa dentre as fases folicular e ovulatória, não ocorrendo influência em seu desempenho físico, mantendo uma constância nos níveis de força de membros superiores, potência de membros inferiores, e como também, as variáveis da composição corporal. Conclui-se que, nas atletas de alto rendimento, pelo fato de diariamente conviver com esforço físico de alta intensidade, pode ocorrer adaptações fisiológicas aos estímulos, contribuindo e permitindo, assim, para uma programação de treino contínua para ambas as fases, sem que haja interrupções em questões das oscilações hormonais durante 0 ciclo menstrual.

Tal resultado será de grande relevância para corroborar com 0 muscular, o que não acontece para a força isométrica, afirmando que a fase menstrual apresenta maiores sintomas que podem influenciar no desempenho e treinamento esportivo (BENTO, 2016).

O resultado da força dinâmica em membros inferiores obteve força muscular superior na fase pós-menstrual, não semelhante ao nosso, não apontou efeitos de alterações na força explosiva (potência) em nenhuma das fases, o que pode ter ocorrido uma familiarização entre os aparelhos e respostas aos estímulos que fora feito em quatro momentos em dois meses. Já na força de membros superiores, os resultados são semelhantes a esta pesquisa, não havendo diferença na força isométrica em membros superiores.

conhecimento científico cujo tal torne-se público contribuindo também com a comunidade acadêmica para o crescimento do curso da área de Educação Física e, de total valor significativo, que servirá de auxílio para que os técnicos possam melhor desenvolver e planejar o treinamento para suas equipes e ao mesmo tempo em que as participantes sejam beneficiadas com os resultados adquiridos, para fins de um bom desempenho dentro do ciclo menstrual em todas as fases.

Não tivemos neste estudo o poder de analisar bioquimicamente as taxas hormonais, qual tipo de estímulos, métodos de programações de treinamento das atletas. Sugerimos um estudo mais aprofundado em termos bioquímicos, nutricionais, para obter dados mais delineados e assim contribuir para as modalidades de alto rendimento do sexo feminino.

\section{REFERÊNCIAS}

ASHLEY, C. D.; KRAMER, M. L.; BISHOP, P. Estrogen and Substrate Metabolism. Sports Medicine, v. 29 , n. 4 , p. $221-227.2000$.

ALEXANDRA M. D.; ZSUZSANA J. B.; ELIEZER B.; ANTÔNIO C. L.; MAURO V. Incidência da Síndrome Pré-menstrual na pratica de Esportes. Revista Brasileira Medica no exercício e no Esporte. São Paulo, v. 15, n. 5, p. 300-331, 2009.

BENTO T. N. Avaliação dos níveis de força muscular em diferentes fases do ciclo menstrual. Florianópolis, 2016. TCC - Educação Física - Bacharelado. Universidade Federal de Santa Catarina - SC. $52 f .2016$.

CONSTANZO, L. S. Fisiologia. 5.ed. Rio de Janeiro: Guanabara Koogan, p. 294. 2013.

CONSTANTINI, N. W. et al. The menstrual cycle and sport performance. Clinics in Sports Medecine., Philadelphia, v. 24, n. 2, p. 51-82. 2005. 
DIAS, I.; SIMÃO, R.; NOVAES, J. S.; Efeito das diferentes fases do ciclo menstrual em um Teste de 10RM. Fitness \& Performance Journal, v. 4, n. 5, p. 291, 2005.

DRUCIAK, C. Análise comparativa dos níveis de força em mulheres durante as fases menstrual e ovulatória. 2015. 41f. Monografia (Bacharelado em Educação Física) - Curso Bacharelado em Educação Física da Universidade Tecnológica Federal do Paraná. Curitiba, p. 32. 2015.

FORTES, L. S.; MORAES, E. M; TEIXEIRA, A. L. S; DIAS, I.; SIMÃO, R. Influência do ciclo menstrual na força muscular e percepção subjetiva do esforço em atletas de natação que utilizam contraceptivos. Revista Brasileira de Ciência e Movimento. Rio de janeiro.;v. 23, n. 3, p. 81-87, 2015.

FLECK, S. J.; KRAEMER, W. J. Fundamentos do Treinamento de Força Muscular. $3^{a}$ Ed. Porto Alegre. Artmed, 2006.

FREITAS, F.; MENKE, C. H. Rotinas em Ginecologia. Porto Alegre: Artmed, 2001.

LIMA. R. C. O.; SANTOS M. Q.; VEIGA P. H. A.; OLIVEIRA M. N. M. Análise da força muscular de preensão manual durante e após o ciclo menstrual. Revista Fisioterapia \& Saúde Funcional. Fortaleza, v. 1, n.1, p. 22-27, 2012.

JEFFERSON C. C., NATÁLIA A. G., DEBORA D. F. M. R., ALEXANDRE G. S.. A influência do ciclo menstrual no desempenho de atletas de futebol feminino. Revista Eletrônica Saúde e Ciência Santos. São Paulo. v. 4. n. 01, p. 39-43, 2014.

JONGE, X. A. J. Effects of the menstrual cycle on exercise performance. Sports Medicine., Auckland, v. 33, n. 11, p. 833-851, 2003.

JUNIOR, A. T. C.; CUNHA A. C. P. T.; SHENEIDER A. T.; DANTAS P. M. S.; Características dermatoglíficas, somatotípicas, psicológicas e fisiológicas da seleção brasileira feminina adulta de handebol. Fitness \& Performance Journal Rio de janeiro, v. 5, n, 02, p. 82, 2006.

KRAEMER, W. J.; ADAMS, K.; CAFARELLI, E.; DUDLEY, G. A.; DOOLY, C.; FEIGENBAUM, M. S.; FLECK, S. J.; FRANKLIN, B.; FRY, A. C.; HOFFMAN, J. R.; NEWTON, R. U.; POTTEIGER, J.8; STONE, M. H.; RATAMESS, N. A. American College of Sports Medicine position stand: progression models in resistance training for healthy adults. Medicine and Science in Sports and Exercise. Madison, v. 41, n. 3, p. 687-708, 2009.

KRAEMER, W. J.; RATAMESS, N. A. Fundamentals of Resistance Training: Progression and Exercise Prescription. Medicine and Science in Sports and Exercise, Madison, v. 36, n. 4, p. 647688, 2004.

LEBRUN, C. M.; RUMBALL, J. S. Relationship between athletic performance and menstrual cycle. Current Women's Health Reports, v.1, p. 232-240, 2001.

MCARDLE ,W. D.; KATCH, F.I.;KATCH, V. L.; Fisiologia: nutrição, energia e desempenho humano. Rio de janeiro. Guanabara Koogan. 8 ed. p. 758, 2016.

MORAES, A. C. F, OLIVEIRA, H. G., MOLENA C. A.F, FULAZ C. S, Relação entre ciclo menstrual e planejamento dos treinos: um estudo de caso. Acta Scientiarum Health Sciencies. Maringá, v. 30, n.1. p.7-11. 2008.

NAAMA W.; CONSTANTINI, M. D., GAL DUBNOV, M. D,; CONSTANCE M.; LEBRUN, M.D. C. M. The Menstrual Cycle and Sport Performance. Clinics in Sports Medicine, v. 24, n. 2, p. 53, 2005.

POWERS, S. K. Fisiologia do exercício: teoria e aplicação ao condicionamento e ao desempenho. Barueri. Manole. 8ed. 2014. 
REDMAN, L. M.; WEATHERBY, R. P. Measuring performance during the menstrual cycle: A model using oral contraceptives. Physical Fitness and Performance, p. 130-136, 2003.

TEIXEIRA A. L.S., JÚNIOR,W. F., MORAES, E. M., ALVES,H. B. DIAS, M.R., Efeito das diferentes fases do ciclo menstrual na composição corporal de universitárias. Revista Brasileira de Prescrição e Fisiologia do Exercício, São Paulo, v. 6, n. 35, p. 428-432, 2012

VALADARES, G. C.; FERREIRA, L. V.; FILHO, H. C.; ROMANO-SILVA, M. A. Transtorno disfórico pré-menstrual revisão - conceito, história, epidemiologia e etiologia. Revista Psiquiatria Clínica, v.33, n. 3, p. 117-123, 2006. 